\title{
À LA RECHERCHE DES AMIS PERDUS : LE BOUQUET DE BOHĖME DE ROLAND DORGELÈS COMME LIVRE-TOMBEAU
}

Roland Dorgelès est connu du large public avant tout comme auteur des Croix de bois, roman-témoignage où il relate ses expériences des tranchées de la Grande Guerre $^{1}$. Or, on ne peut pas oublier qu'il est aussi auteur emblématique du XVIII ${ }^{\mathrm{e}}$ arrondissement parisien. Venu d'Amiens, il est arrivé à Montmartre en 1903 à l'âge de 18 ans et s'est proclamé aussitôt montmartrois, traitant dès lors le quartier comme sa nouvelle patrie ${ }^{2}$. Il a manifesté son attachement à Montmartre dans de nombreux récits tant fictifs (Le Château des brouillards, 1932) que factuels, ceuxci recouvrant la forme de souvenirs (Quand j'étais montmartrois, 1936 ; Bouquet de bohème, 1947 ; Au beau temps de la Butte, 1949 ; Portraits sans retouches, 1952). Il n'était d'ailleurs pas le seul à faire de Montmartre le sujet de prédilection de son écriture. Sans en avoir l'intention, il a formé avec Francis Carco et Pierre Mac Orlan un groupe informel d'écrivains qu'Armand Lanoux propose d'appeler «l'école de Montmartre»:

On peut être surpris que l'appellation « école de Montmartre »n'ait pas été utilisée pour qualifier un mouvement littéraire né avec le siècle et éteint en 1973 par la mort de son principal protagoniste Roland Dorgelès. [...] Trois écrivains, proches d'inspiration et de même attirance quasi physique pour une colline excentrique, ont marqué de leur griffe un moment de la sensibilité littéraire d'une France parisianisée, corsetée depuis ses femmes jusqu'à son tout neuf métro ${ }^{3}$.

${ }^{1}$ R. Trouilleux, Montmartre des écrivains, Bernard Giovanangeli Éditeur, Paris 2005, p. 151.

2 Ibidem, p. 142.

3 A. Lanoux, "Trois personnages en quête d'une bohème ", Magazine Littéraire 185, 1982, p. 16. 
Nicolas Hewitt, quant à lui, tout en remarquant également le vent d'inspiration qui souffle du Montmartre de la Belle Époque, propose dans son article une perspective sociologique et affirme que la popularité du sujet relève moins d'une vraie fascination que d'une mode permettant de profiter des clichés culturels bon marché ${ }^{4}$.

Hewitt adopte une attitude distanciée vis-à-vis de ces récits : selon lui leurs auteurs se caractériseraient par une grande dose de narcissisme et une forte tendance à embellir la vie de l'avant-guerre à Montmartre ${ }^{5}$. Roland Dorgelès n'échappe pas à la critique du chercheur : l'auteur du Château vendrait au public une image rosée de la Butte et idéaliserait la vie du village montmartrois ${ }^{6}$. Effectivement on peut avoir l'impression que selon Dorgelès, la vie à Montmartre ressemblerait à un âge d'or perdu auquel il voudrait revenir à tout prix. Il semble que le fonctionnement de ce type de discours ne relève que d'une simple mode voulant vendre une image idéalisée de la Butte. D'ailleurs, l'auteur lui-même dit ouvertement qu'il ne prétend nullement donner une image vraie de sa " patrie» :

Ainsi, je viens à peine de commencer ma confession et déjà les scrupules m'envahissent. Je m'interroge, je doute de moi... Était-il tellement vaste, le parc de la Belle-Gabrielle où nous donnions nos rendez-vous ? [...] J'hésite... Je ne sais plus... Le Temps, en vieux restaurateur qui connaît son métier, repeint les personnages et rafraîchit le décor. Quand la toile était neuve, c’était peut-être moins beau (p. 35) ${ }^{7}$.

Il nous semble plus justifié de voir dans les souvenirs de Dorgelès l'expression d'un thème récurrent dans son œuvre, beaucoup plus profond, à savoir la hantise de l'oubli $^{8}$. Précisons tout d'abord que les souvenirs de Dorgelès ont une structure épisodique, la narration n'est soumise à aucun ordre chronologique ni logique, l'auteur raconte de nombreuses anecdotes présentant les « exploits » de ses amis montmartrois. Or l'image de ces aventures montmartroises n'est pas aussi

${ }^{4}$ «Throughout the inter-war years, writers who had not become successful until after the War and who had largely done so through their depiction of their own apprenticeship in Montmartre's Bohemia, tended to exploit pre-war Montmartre as both locus and subject-matter, much in the same way that Utrillo, in his later career, exploited it. Significantly, one of the most characteristic genres of Montmartre writing of the inter-war period is the memoir, either fictional or non-fictional, with multiple examples from Dorgelès, Carco, Mac Orlan, and André Warnod, and other contributions from writers such as Paul Yaki, Géo Cim, Jean Vertex, Georges Charensol, J.-P. Crespelle, André Salmon and others » (N. Hewitt, « Images of Montmartre In French Writing 1920-1960: la bohème réactionnaire », French Cultural Studies 4:129, 1993, p. 132).

5 Ibidem, p. 131.

6 « Roland Dorgelès, for example, is characterized in all of his Montmartre writing by a limited and essentially rosy view of the Butte itself » (ibidem, 131). À ce sujet, voir aussi la page 135.

${ }^{7}$ Les numéros de pages entre parenthèses qui suivent les exemples renvoient à R. Dorgelès, Bouquet de bohème, Albin Michel, Paris 1947.

${ }^{8}$ Elle est fortement présente surtout dans son œuvre parlant de la Grande Guerre. $C f$. C. Trevisan, Les fables du deuil. La Grande Guerre : mort et écriture, Presses Universitaires de France, Paris 2001, pp. 178-179. 
positive que le voudrait Hewitt, comme le montre l'exemple (il y en a beaucoup d'autres) cité ci-dessous :

Il y a trop d'injustices dans cette distribution de bienfaits. Si je pouvais, dans l'Au-delà, choisir mon poste, j'ouvrirais une boutique de raccommodeur, où je réparerais les bévues du destin. Alors, un Juan Gris ne finirait pas obscurément à l'hôpital et Jacques Vaillant ne se ferait pas sauter la cervelle. (p. 180)

Dorgelès recourt à plusieurs reprises au topos de la Fortune injuste qui a mené de nombreux artistes à une mort prématurée, très souvent dépourvue d'un rituel funéraire qui permettrait d'accomplir le deuil. Ainsi proposons-nous de traiter Bouquet de bohème comme une sorte de discours funèbre à travers lequel l'auteur essaye de ressusciter la mémoire de ses amis pour leur offrir un substitut de funérailles dignes de leur talent. Vu dans cette perspective, les souvenirs de Dorgelès ressembleraient aux récits de la Grande Guerre et pourraient correspondre à la réflexion de Carine Trevisan. Selon Trevisan, une grande partie de ses fictions de guerre sont issues du traumatisme de la mort anonyme des tranchées ces et du deuil non accompli qui l'a accompagnée :

L'écriture peut être conçue comme le prolongement de la sépulture, le premier geste, avec l'acte des funérailles de la symbolisation de la mort. [...] nombreuses sont les procédures par lesquelles le texte accomplit symboliquement les gestes des funérailles et se dit, se veut, tombeau, ressemblant et conservant dans son espace clos les restes du mort ${ }^{9}$.

L'un de ces procédés dont parle Trevisan consiste en un travail discursif de singularisation qui permet de discerner dans la masse anonyme des poilus des hommes en chair et en os, avec toutes leurs émotions, espérances et douleurs. « Il s'agit de rendre au mort un visage, une histoire ${ }^{10}$. On peut remarquer le même procédé chez Dorgelès, qui à travers de nombreuses anecdotes concentrées autour des personnages montmartrois, restitue leur histoire et par là, leur individualité. Chez Dorgelès, ce travail de singularisation se fait à un double niveau : celui des individus et celui du groupe. Dans la suite de notre article nous essayerons de montrer les démarches à travers lesquelles l'auteur des Croix des bois cherche à donner ce trait d'individualité tant à ces amis qu'à toute la collectivité montmartroise.

Comme nous l'avons déjà signalé, les souvenirs de Dorgelès se concentrent autour de personnages avec lesquels il était lié par des liens d'amitié. Or, il faut souligner que la notion d'amitié, qu'il emploie comme synonyme de la notion connexe de camaraderie, est un trait distinctif et, par là, identitaire de la bohème du XVIII ${ }^{\mathrm{e}}$ arrondissement :

Pourtant, sur un point, je suis certain de ne pas me tromper : Montmartre était la patrie de la camaraderie. Dans notre clan, tout se mettait en commun, à commencer par l'espoir. Nous étions fiers les uns des autres. (p. 35)

\footnotetext{
${ }^{9}$ Ibidem, p. 117.

${ }^{10}$ Ibidem, p. 179.
} 
L'idée de liens de solidarité forts est renforcée par l'emploi fréquent de mots tels que clan, confrérie, phalanstère, ce qui suggère aussi une certaine fermeture et une distance vis-à-vis des nouveaux venus. On en trouvera un bon exemple dans la description de la première visite de Pierre Mac Orlan au Lapin Agile, où il a été obligé de s'attirer les bonnes grâces des habitués du cabaret :

Francis Carco, qui habitait la rive gauche nous rejoignit rue des Saules un soir d'hiver, amené par un ami, car il fallait être présenté, comme au Jockey-Club. [...] il nous apparut le sourire aux lèvres, plein d'assurance. Très correctement mis : veston noir, haute cravate à la Rostand, et bottines vernies. Rien de comparable, on le voit, avec ses personnages. À cause des vernis, certains restaient sur la réserve : les rapins ne prisaient pas les mises trop soignées. Le nouveau dut le sentir. Il demanda au patron de reprendre sa guitare, grimpa d'un bond sur la table et se mit à chanter un refrain de Marseille, coloré de l'accent du Vieux Port. Aussitôt, les visages se détendirent. Rien qu'à l'entendre, on se prenait à l'aimer. [...] quand il redescendit toutes les mains se tendirent. "Comment t'appelles-tu ? Tu es de Paris ? Assieds-toi là !... Qu'est-ce que tu prends ? » La bande du Lapin l'avait adopté. (p. 26)

Comme le montre l'exemple cité, la première étape du « rituel » d'admission au groupe se fait en fonction du critère vestimentaire, ce qui, dans le cas Pierre Mac Orlan, constituait plutôt une entrave dont il a su se débarrasser par son talent scénique. L'aspect extérieur des personnes constitue chez Dorgelès l'élément essentiel de leur description, que l'auteur s'abstient toutefois de détailler longuement et de manière réaliste, se limitant à quelques éléments typiques de leur tenue. Ainsi, Pablo Picasso se distingue par sa veste de toile bleue, Aristide Bruant par son foulard rouge et son feutre, Maurice Vlaminck par son chandail à col roulé, Max Jacob par son caban soutaché de rouge, et Kees van Dongen par ses espadrilles. Francisque Poulbot et sa femme, quant à eux, sont associés aux habits de noces (« lui en habit noir, elle fleurie d'orangers », p. 15) en raison des mariages théâtralisés qu'ils organisaient chaque année pour divertir leurs amis montmartrois. Dorgelès évoque aussi ses propres attributs, à savoir le gilet rouge et la fameuse frange qui le distinguaient de ses confrères. Cette auto-description joue d'ailleurs un double rôle dans le récit, car Dorgelès, tout en soulignant par ce petit détail son individualité, tient aussi à montrer son appartenance à la collectivité montmartroise, ce qui est renforcé dans le discours par l'emploi fréquent des pronoms «nous » et « on ».

Hormis les descriptions vestimentaires qui jouent un rôle important dans l'individuation des personnages, Dorgelès décrit aussi des appartements qui témoignent du caractère exceptionnel de ses amis. On peut donner comme exemples les descriptions de l'appartement de Max Jacob, qui trahit son penchant pour le mysticisme et les sciences occultes :

Ce désordre avait même gagné les murs, où les signes du zodiaque se mêlaient à des maximes bizarres et des adresses de copains. (Bientôt, il devait y ajouter un cercle à la craie bleue, pour marquer l'emplacement où la Sainte Face du Christ lui était apparue, vision qui entraîna sa conversion.). (p. 116)

et de l'appartement d'André Salmon, qui surprenait ses invités par son engouement pour les horloges. Il en avait six : 
Salmon n'étant pas riche, n'avait pas de meubles, nul ne s'en étonnait ; faute de chaises, on s'asseyait à la turque sur un tapis de Boukhara, qu'il avait acheté à Nijni-Novogrod, lorsqu'il était élève consul, mais ce qui surprenait les visiteurs, c'était de voir, le long des murs, six grandes horloges franc-comtoises, qui marquaient toutes des heures différentes.

- Il est trois heures et demie à Saint-Pétersbourg, annonçait imperturbablement le maître de maison en consultant les cadrans. Minuit moins le quart à Lisbonne... Sept heures vingt à Rio-de-Janeiro. (p. 281)

Pourtant, l'objet le plus incongru se trouvait dans le salon apparemment peu original d'un musicien amateur dont Dorgelès ne souvient même pas du nom :

À première vue il n'avait rien d'original et son appartement, bourgeoisement meublé, ne dénotait aucune fantaisie, mais si l'on poussait la porte du salon [...] on apercevait un objet qui modifiait aussitôt votre jugement : sur la cheminée, en guise de pendule, une tête de mort. Une vraie... (p. 296)

Dorgelès raconte par la suite l'histoire du propriétaire de l'appartement qui a fait un voyage en Afrique en compagnie d'un ami. Celui-ci, suite à une maladie, est mort « en pleine brousse équatoriale ». Le musicien, désemparé mais ne voulant cependant pas abandonner le corps de son ami au cœur de la forêt africaine, a décidé de le décapiter et de faire sécher la tête afin de pouvoir la rendre à la famille du défunt en guise de dépouille. Mais la famille, horrifiée par ce geste, n'a pas apprécié le sacrifice du musicien et a refusé l'objet qui est ainsi devenu un élément décoratif.

Si les exemples cités ci-dessus s'inscrivent dans les procédés d'individuation de personnages particuliers, on peut observer également une forte tendance à ressusciter l'histoire collective de toute « la confrérie » montmartroise. Elle se manifeste surtout par des anecdotes témoignant de l'esprit fantasque et du goût des artistes pour les blagues. Le canular avec la participation de Lolo, l'âne-mascotte du Lapin Agile devenu « peintre » et auteur du tableau « Et le soleil se coucha sur l'Adriatique ", est entrée dans la légende ${ }^{11}$, mais il y en a d'autres, moins connues, qui se résument le plus souvent à un même scénario : enfreindre les usages sociaux établis et, si l'occasion se présente, arnaquer un fournisseur ou un restaurateur pour se remplir l'estomac sans bourse délier. Dans la plupart des cas, il s'agissait d'actes individuels à caractère délictueux, mais il arrivait aussi que certains artistes aient affaire aux représentants de l'ordre en raison de leur naïveté. Tel est par exemple le cas de Pierre Mac Orlan, dessinateur habile et bon observateur, qui s'est retrouvé complice d'une bande de dévaliseurs d'églises en croyant produire des esquisses pour un livre d'art. Après avoir découvert la véritable destination de ses croquis, il a été pris de panique et a même pensé fuir Paris :

Pendant des semaines, il ne dormit plus, frémissant au petit jour lorsque des pas ébranlaient l'escalier. Mais le crocheteur de tabernacles était, à sa façon, un homme d'honneur et, fidèle à la « loi du milieu », ne livra pas le nom de son jeune assistant. Mac Orlan, par la suite, a merveilleusement décrit les transes du hors-la-loi que traque la police. Il savait de quoi il parlait... (pp. 77-78)

${ }^{11}$ Voir à ce propos J. Seigel, Paris bohème 1830-1930, Gallimard, Paris 1991, pp. 326-327. 
Pourtant, dans les extravagances décrites par Dorgelès, il s'agissait le plus souvent de transformer la vie quotidienne de Montmartre en spectacle. Comme le remarque Jerrold Seigel : « La bohème était donc une forme de théâtre social, dont le public, ostensiblement hostile, avait un obscur besoin $»^{12}$. On peut traiter les boutades montmartroises décrites par Dorgelès comme un écho des processions de la Vachalcade, autrement appelée la Fête de la Vache enragée : un festival organisé à Montmartre par Adolphe Wilette en 1896 et 1897 pour parodier la célébration de la fête municipale du Bœuf Gras ${ }^{13}$. La fête, qui a réuni un nombre considérable d'anarchistes et de socialistes, s'est inscrite largement dans le contexte politique et social de la lutte contre le gouvernement de la Troisième République. Toutefois, pour les artistes montmartrois, il s'agissait surtout de manifester leur opposition culturelle et spirituelle à la société bourgeoise de la fin du siècle et, par là, d'entretenir une identité collective intrinsèquement liée à l'espace du XVIII ${ }^{\mathrm{e}}$ arrondissement ${ }^{14}$. Bien que la Fête de la Vache enragée ait connu un grand succès, elle n'a pas pu continuer faute de fonds. Les anecdotes évoquées par Dorgelès témoignent tout de même que le besoin de manifester l'identité collective à travers des coups de théâtre spontanés a longtemps persisté au sein de la communauté montmartroise.

$\mathrm{Au}$ niveau du récit, ces anecdotes contribuent effectivement à créer l'effet d'une atmosphère bucolique et insouciante de la vie bohème à Montmartre à la charnière des siècles, démarche que Hewitt ${ }^{15}$ voit d'un œil critique. Or, si nous nous détachons de l'univers représenté par les souvenirs pour examiner le cadre dans lequel s'inscrit la narration, on découvrira un contraste de tonalité remarquable. Dorgelès-narrateur place son récit dans le champs métaphorique d'une promenade solitaire à travers un village hanté par les revenants. Ceux-ci sont en majeure partie ses amis montmartrois. L'un des passages qui symbolise le mieux cette rencontre avec le monde de l'au-delà fait référence aux tableaux d'Utrillo où, employant la démarche de la mise en abîme, le narrateur « découvre » derrière l'espace montmartrois apparemment vide un monde peuplé de fantômes ${ }^{16}$ :

Alors, le long des murs, je vois glisser des ombres ; Modigliani, droit comme un jeune prince, Chaudois, le taciturne enveloppé dans son long pardessus, Max Jacob, l'œil étincelant derrière le monocle, Frédé menant son âne, Depaquit coiffé de son gibus de cérémonie, Marie Vizier, le balai à la main, Sagot-le-Fou une toile sous le bras, puis, la dernière, Suzanne Valadon, mince et vive, suivie d'un grand diable aux gestes disloqués...

12 Ibidem, p. 323.

${ }^{13}$ V. Datta, «A Bohemian Festival; La Fête de la Vache Enragée », Journal of Contemporary History 2, 1993, p. 195.

${ }^{14}$ Comme le remarque Datta, Montmartre était traité par ses habitants comme une sorte d'espace sacré et le festival leur a fourni la possibilité de manifester une solidarité sociale qui s'est constituée dans le cadre de cet espace symbolique (ibidem, p. 205).

15 N. Hewitt, op. cit.

${ }^{16}$ Remarquons à l'occasion que ce passage réalise, comme sous un verre grossissant, la technique que nous avons évoquée plus haut et qui consiste à individualiser chaque personne au moyen d'un attribut caractéristique. 
Comment avons-nous pu croire que les rues d'Utrillo étaient désertes ? elles sont peuplées de fantômes. Mais, pour les découvrir, il fallait que le temps eût passé. (p. 227)

À côté des artistes dont il est capable de citer le nom, Dorgelès évoque à plusieurs reprises des artistes anonymes qui sont disparus sans laisser de traces ni matérielles, sous la forme d'une pierre tombale, ni mentales, dans la mémoire de ceux qui les ont suivis. Il se représente en même temps comme l'un des survivants, chargé de porter témoignage non seulement des joies de la vie bohème, mais aussi de ses dangers et sacrifices :

Nous seuls, leurs camarades, entretiendrions leur souvenir ; nous seuls, surtout, pourrions disputer un instant à l'oubli le nom des malchanceux qui ne laissaient même pas une œuvre pour survivre. Ils avaient sacrifié plus que d'autres, ces peintres sans renom, ces écrivains obscurs, car ils renonçaient au bonheur de créer, car ils offraient en holocauste ce qu'ils ne tenaient pas encore, mais qui leur était promis entre les mains des Dieux.

C'est en pensant à eux que j'ai repris le chemin de Montmartre, espérant y retrouver leurs ombres. (pp. 348-349)

Les souvenirs deviennent ainsi une sorte du tombeau mental dont les artistes ont été privés. Le titre Bouquet de bohème, vu dans cette perspective, se dote d'un double sens : d'un côté « le bouquet» symbolise toute la jeunesse montmartroise, mais en même temps, il fait penser à la gerbe funéraire qu'on devrait déposer sur sa tombe. Cette portée symbolique accordée aux fleurs est d'ailleurs annoncée tout au début du récit : " Cher bouquet de jeunesse, flétri, poussiéreux, desséché... » (p. 9).

De cette manière le titre annonce et résume toute la profondeur oxymoronique des souvenirs de Dorgelès, qui tout en réveillant «l'image rosée » d'une vie en plein épanouissement, charrie sournoisement son épitaphe.

\section{IN SEARCH OF LOST FRIENDS : ROLAND DORGELÈS' BOUQUET DE BOHEME AS A SEPULCHRE BOOK}

\section{Summary}

The article is a reflection on Roland Dorgeles' Bouquet de bohème depicting the period of his life in Montmartre at the beginning of the $20^{\text {th }}$ century. We try to demonstrate that behind the apparently idyllic image of the XVIII arrondissement, Dorgelès presents a much more nostalgic and pessimistic vision of the bohemian life, which for many artists ended with an anonymous death in their heyday. Through series of anecdotes and hilarious details, he intends to create a discursive tombstone dedicated to the memory of his young friends who disappeared too soon.

Key words: bohemianism, epitaph, friendship, memory, Montmartre. 